Monatsschr Kinderheilkd 2018 $\cdot 166: 3-5$ https://doi.org/10.1007/s00112-017-0424-8 Online publiziert: 15. Dezember 2017

(c) Springer Medizin Verlag GmbH, ein Teil von Springer Nature 2017

CrossMark

\title{
D. Karall
}

Department für Kinder- und Jugendheilkunde, Klinik für Pädiatrie I, Spezialbereich Angeborene Stoffwechselstörungen, Medizinische Universität Innsbruck, Innsbruck, Österreich

\section{Mit neuen Vorzeichen auf bewährten Wegen}

\section{Zur ÖGKJ-Präsidentschaft 2018 bis 2020}

spannen muss, zwischen „Seltenem und Häufigem“, also einerseits der hochspezialisierten Medizin und andererseits der primären Versorgung im niedergelassenen Bereich der Altersgruppe der 0- bis 18-Jährigen. Beide Bereiche sind integraler Bestandteil der Agenden der ÖGKJ - und sind für mich wie die beiden Seiten einer Münze, die sehr unterschiedlich sein können und trotzdem Teil des Ganzen sind.

Es ist mir eine Ehre und Freude, dass ich ab dem 01.01.2018 von meinem Vorgänger Prof. Dr. Wolfgang Sperl die Präsidentschaft der Österreichischen Gesellschaft für Kinder- und Jugendheilkunde (ÖGKJ) übernehmen werde.

Für die (ÖGKJ) ist es gewissermaßen ein historischer Moment - es ist das erste Mal in ihrer 55-jährigen Geschichte, dass eine Frau die Position der Präsidentschaft bekleidet, und es ist gleichzeitig auch das erste Mal, dass diese Position nicht der Leiter einer Klinik für Pädiatrie oder der Leiter einer klinisch pädiatrischen Abteilung innehat.

Mit der ÖGKJ verbinden mich seit den Anfängen meiner Ausbildung wichtige Meilensteine. Im Rahmen der Mitarbeit bei der Ausrichtung der Jahrestagungen in Innsbruck (1995) und Mayrhofen im Zillertal (1996) habe ich bald erlebt, welche Kerninhalte die Gesellschaft prägen. Auch in der Gegenwart und der Zukunft ist die Ausrichtung von Fortbildungen eine wesentliche Säule der ÖGKJ.

\section{Persönlicher Werdegang}

Mein persönlicher Werdegang beruht auf 2 Schwerpunktthemen - und ich denke, dass diese gut die Dichotomie innerhalb der ÖGKJ widerspiegeln, die den Bogen

\section{\) Den Bogen spannen zwischen hochspezialisierter Medizin und Primärversorgung}

Von Anbeginn meiner Ausbildung war ich in die Thematik rund um ,angeborene Stoffwechselstörungen “ und damit seltene Krankheiten eingebunden. Von Wolfgang Sperl habe ich den Bereich "angeborene Stoffwechselstörungen“" in Innsbruck übernommen, als er sich 1997 der Leitung der Kinderklinik in Salzburg widmete. Fasziniert - und bis heute in dem Bereich tätig - staune ich noch immer darüber, wie viel aus gestörten Stoffwechselwegen über die physiologischen Prozesse gelernt werden kann. Dabei habe ich immer die enge nationale (und auch internationale) Vernetzung zwischen "gleichgesinnten“ Kollegen geschätzt. Ich bin überzeugt, dass Vernetzung und persönlicher Austausch mit Kollegen die Mittel sind, mit denen wir den z. T. sehr unterschiedlichen Bedürfnissen von Kindern, Jugendlichen sowie deren Eltern und Familien am besten begegnen. In einem starken Netzwerk - und das hat jede(r) von uns seinen Anforderungen und Bedürfnissen entsprechend aufgebaut - sind wir besser ausgerüstet, mit den Herausforderungen des Alltags umzugehen.

Etwa die Hälfte der angeborenen Stoffwechselstörungen, die wir derzeit diagnostizieren, tritt im Neugeborenenalter auf. Und etwa bei der Hälfte der angeborenen Stoffwechselstörungen, die wir diagnostizieren, besteht ein wesentlicher Teil oder die Therapie überhaupt aus einer „Diät“ - also Modifikation der Ernährung in irgendeiner Weise. Daher erachte ich den Begriff „Ernährungstherapie“ als günstiger, da er umfassender und klarer ist als der Begriff „Diät“, der eher an die Einschränkungen als die Möglichkeiten denken lässt. Außerdem beinhaltet „Ernährungstherapie“, dass es sich tatsächlich bei der Modifikation der Ernährung um eine essenzielle Säule in der Behandlung von Patienten handelt.

Ich war somit bald auch mit dem Themenbereich , altersentsprechende Ernährung von Anfang an" konfrontiert. Auch in diesem Bereich habe ich von der Beschäftigung mit dem Seltenen vieles gelernt. Als Pädiater stehen wir ganz am Anfang des Lebens eines Menschen heute bin ich davon überzeugt, dass neben dem Impfwesen die gute Etablierung einer sinnvollen Ernährung (Stichwort „Förderung von Stillen“) die beiden Säulen sind, die uns in der Prävention von Erkrankungen weltweit in puncto Erhöhung der Lebenserwartung und Verbesserung der Lebensqualität durch Reduktion von Mortalität und Morbidität am meisten weitergebracht haben und weiterbringen werden.

Die Aufgaben, die wir als Ärzte für Kinder- und Jugendheilkunde haben, sind vielfältig wie wohl in keiner ande- 
ren medizinischen Disziplin. Wir decken nicht nur alle Organe $a b$, sondern erfüllen diese Aufgabe auch über einen Zeitraum von 18 Jahren, in dem viel an Entwicklung und Veränderung der Betreuten passiert. Das heißt, unser fachliches Wissen ist groß und meist breit. Gleichzeitig sollen wir oft dem Anspruch gerecht werden, alles ,jetzt sofort, gut und schnell“ zu lösen. Ärzte für Kinder- und Jugendheilkunde sind also auch sehr flexibel.

Dennoch haben wir teilweise $\mathrm{zu}$ kämpfen, mit dem Image, die „kleinen“ Kollegen für die „kleinen“ Patienten zu sein ... Aber nicht umsonst vertrauen uns Eltern ihre Kinder an.

\section{Entwicklung der Öster- reichischen Gesellschaft für Kinder- und Jugendheilkunde}

Die ÖGKJ ist keine kleine Gesellschaft „kein kleiner Haufen“ - mehr. Die Vernetzung über die ÖGKJ als ein offizieller Zusammenschluss des Berufsverbandes ist in diesem Sinne nicht zu unterschätzen. Auch wenn der persönliche Mehrwert u. U. nicht sofort ersichtlich ist, möchte ich doch dazu ermutigen, sich der Gesellschaft anzuschließen. Die ÖGKJ hat inzwischen über 1600 Mitglieder. Sie ist in ihrer Struktur über mehrere Jahrzehnte gewachsen, sodass das neue Präsidium aufbewährten Strukturen aufund weiterbauen kann. Das schätze ich sehr. Gleichzeitig baue ich auf jede(n) Einzelne(n) - jede(r) hat etwas beizutragen - und nur gemeinsam sind wir stark.

Ich übernehme von meinem Vorgänger Prof. Dr. Wolfgang Sperl eine gut geführte Gesellschaft, die in den letzten Jahren etliche Meilensteine gesetzt hat. Diese sind z. B. Präsenz, Beratung, Ansprechpartnerschaft in Fragen der Kinder- und Jugendgesundheit für die Bundesministerien für Gesundheit und Soziales und den Hauptverband der Sozialversicherungsträger; adäquate Positionierung der Pädiatrie in Ausbildungsfragen; Sicherstellung der Spezialisierungen in der Pädiatrie; Ausrollung der Rehabilitation für Kinder und Jugendliche in Österreich; Vernetzung mit verwandten Gesellschaften wie der Deutschen Ge- sellschaft für Kinder- und Jugendmedizin [DGKJ]; weitere Einbeziehung u.a. der politischen Kindermedizin und der Kinderliga.

Es ist mir ein Anliegen, die Kontinuität in den Agenden der ÖGKJ zu wahren, d.h., bewährte Teams weiter in den bereits laufenden Agenden wirken zu lassen sowie bereits bestehende Kontakte zu Entscheidungsträgern zu pflegen.

Dabei sehr wertvoll und hilfreich ist die Tatsache, dass die ÖGKJ mit Beginn des Jahres 2018 über einen Geschäftsführer verfügt, der über die laufenden Agenden einen Bogen der Kontinuität spannen wird. Wir sind sehr froh, dass wir Prim. Univ.-Prof. Dr. Reinhold Kerbl (Leiter der Abteilung für Kinder und Jugendliche am Landeskrankenhaus Hochsteiermark/Leoben) für diese Position gewinnen konnten.

\section{Zukünftige Themen mit besonderem Gewicht}

Für die nächsten 3 Jahre stehen meiner Meinung nach 3 Bereiche auf der Agenda, denen ich gemeinsam mit meiner geschätzten Kollegin PD Fr. Dr. Scholl-Bürgi (ÖGKJ-Sekretärin für die nächsten 3 Jahre) besonderes Gewicht geben möchte:

Nachwuchsförderung. Der Aspekt der guten Ausbildung und Ausrüstung von Nachfolgern in allen Bereichen der Pädiatrie betrifft selbstverständlich nicht nur unser Fach, sondern zunehmend auch andere Disziplinen. Allerdings ist die Pädiatrie insofern besonders, als sie eigentlich und zunehmend ein ,weibliches" Fach ist (hoher Frauenanteil) und daher der Bedarf an Teilzeitmodellen sowohl für die Ausbildungszeit als auch für die spätere Berufstätigkeit möglicherweise schon früher als in anderen Fächern sichtbar geworden ist. Zunehmend sind wir herausgefordert, die Aspekte, die die jüngere Generation in die medizinische Versorgung hereinbringt, in die Zukunftsplanung des Faches miteinzubauen. Ich erachte Schlagwörter wie ,Generation Y“, ,WorkLife-Balance" nicht nur als Herausforderung, sondern insbesondere auch als Chance, über Modelle nachzudenken und solche zu erarbeiten, die zukünftig und langfristig eine tragfähige medizinische Versorgung von Kindern und Jugendlichen möglichst wohnortnah und flächendeckend gewährleisten.

Öffentlichkeitsarbeit. Öffentlichkeitsarbeit ist für die ÖGKJ nichts Neues, sondern ist schon lange essenzieller Bestandteil innerhalb der Gesellschaft (aktuell: ÖGKJ-Pressestelle bei Ralph Kerschbaumer, studio 12). Allerdings sind wir als in der Medizin Tätige meist nicht damit vertraut, Medien sinnvoll zu nutzen, um unsere Inhalte zu transportieren. Der Trend der Zeit fordert uns, dass wir uns mit den medialen Möglichkeiten auseinandersetzen und sie positiv anwenden. Ziel für mich wäre es, eine Dynamik innerhalb der ÖGKJ zu entwickeln bzw. $\mathrm{zu}$ erreichen, in der die Mitglieder für sie wichtige und verschiedene Themen einbringen, die wir als Gesellschaft unterstützen und verbreiten können.

Vernetzung. Vernetzung ist für mich das Stichwort für eine funktionierende, sich entwickelnde Gemeinschaft („Mit Reden kommen die Leut' zusammen "). In erster Linie gilt die Vernetzung zwischen Kollegen, übergreifend das Alter und die Spezialisierung betreffend. Am besten wird einfach von Vorbildern/Mentoring gelernt, sowohl was die klinische Tätigkeit als auch die wissenschaftliche Arbeit betrifft. Für das Weiterkommen, Themen wie Projektförderung u. Ä. ist die Einbindung in eine Gruppe/ein Netzwerk nicht mehr wegzudenken. In diesem Sinne wünsche ich mir, dass es uns gelingt, den guten Austausch und die bereits bestehenden Netzwerke über die ÖGKJ noch besser sichtbar und damit effektiver zu machen, sowohl bezüglich der klinischen Tätigkeit als auch der wissenschaftlichen Arbeit. Ein Instrument für Austausch und Vernetzung könnte vermehrt auch die ÖGKJ-Homepage werden, die, beginnend in den letzten beiden Jahren, eines „Face liftings” unterzogen wurde, mit dem sie im neuen Jahr online geht (http://www.paediatrie.at). 


\section{Vernetzung}

An dieser Stelle möchte ich abschließend noch betonen, dass wir in Österreich aufgrund der überschaubareren Größe unseres Landes einen Vorteil durch gute Vernetzung genießen. Nicht nur im Bereich der spezialisierten Medizin, sondern auch im Rahmen der einzelnen Disziplinen und der Bereiche der Pädiatrie kennen die Player sich untereinander und sind regional, national sowie auch international gut miteinander vernetzt. Das ist eine sehr gute Voraussetzung für die „State-of-the-art“-Betreuung von $\mathrm{Pa}$ tienten, die wir gut nutzen können, und für die wir von Kollegen aus größeren Ländern der Europäischen Union (EU) durchaus beneidet werden.

Für die gesetzten Vorhaben und Ziele zähle ich weiterhin auf Ihre Unterstützung und gute Zusammenarbeit. Gerne nehme ich Ideen, Vorschläge, Pläne auf!

Herzliche Grüße und beste Wünsche für ein erfolgreiches Jahr 2018

Ihre

A. Univ.-Prof. Dr. Daniela Karall

\section{Korrespondenzadresse}

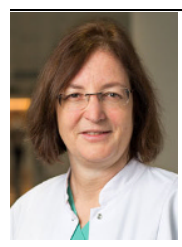

A. Univ.-Prof. Dr. D. Karall

Department für Kinderund Jugendheilkunde, Klinik für Pädiatrie I, Spezialbereich Angeborene Stoffwechselstörungen, Medizinische Universität Innsbruck

Anichstr. 35, 6020 Innsbruck, Österreich

Daniela.Karall@i-med.ac.at

Interessenkonflikt. D. Karall gibt an, dass kein Interessenkonflikt besteht.
C.

Deutsche Gesellschaft für Rheumatologie e.V.

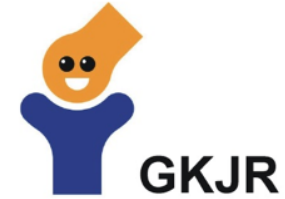

Gesellschaft für Kinder- und Jugend rheumatologie

\section{CALL FOR ABSTRACTS}

\section{Kongress der Deutschen Gesellschaft für Rheumatologie (DGRh)}

zusammen mit

32. Jahrestagung der Deutschen Gesellschaft für Orthopädische Rheumatologie (DGORh),

20. Forum Experimentelle Rheumatologie (FER) und

der Wissenschaftlichen Herbsttagung der Gesellschaft für Kinder- und Jugendrheumatologie (GKJR)

vom 19. bis 22. September 2018, Rosengarten in Mannheim

Liebe Kolleginnen und Kollegen,

wir möchten Sie herzlich einladen, Ihre Abstracts für den 46. Kongress der DGRh einzureichen. Beiträge können zu allen Themenbereichen der klinischen und experimentellen Rheumatologie, der Kinderrheumatologie sowie zu rheumaorthopädischen Fragestellungen und zur Versorgungsforschung eingereicht werden.

Schwerpunkte des diesjährigen Kongresses sind

- RA - vom Labor zur klinischen Anwendung

- Immuntherapie fächerübergreifend

- Seltene Erkrankungen in der Rheumatologie

- Knorpelregeneration und Knorpelreparatur

\section{Ihre Abstracts}

- sollten zwischen 250 und max. 350 Wörter enthalten und in deutscher oder englischer Sprache eingereicht werden - außer für das 20. Forum für Experimentelle Rheumatologie, dort bitte ausschließlich in englischer Sprache,

- werden in einem anonymisierten Verfahren durch das Abstract-Komitee begutachtet,

- können zusätzlich als Vortrag ausgewählt werden,

- können nur über die Internetseite www.dgrh-kongress.de angemeldet werden.

Alle Hinweise zum Prozedere sowie die Regeln zu Inhalt, Gestaltung und Beurteilung finden Sie auf der Homepage unter www.dgrh-kongress.de.

Einsendeschluss ist der 31. März 2018. (Bitte beachten Sie: Diese Frist wird nicht verlängert!)

Wir bitten Sie, diesen Aufruf an interessierte Kolleginnen und Kollegen weiterzuleiten. Das

Vorprogramm wird voraussichtlich im April 2018 im Internet veröffentlicht.

Mit freundlichen Grüßen

Prof. Dr. Chritoph Fiehn, DGRh-Kongresspräsident 2018

Prof. Dr. Bernd Swoboda, DGORh-Kongresspräsident 2018 